\title{
Proposta de um roteiro de apoio à descrição de lesões bucais como instrumentalização para a comunicação profissional
}

\author{
Maria Inês Meurer*; Caroline Zimmermann**; Liliane Janete Grando*
}

\section{RESUMO}

A boa comunicação escrita é uma habilidade essencial para a troca de informações entre profissionais da saúde. No contexto do SUS, essa troca ocorre por meio de documentos de referência e contrarreferência. Considerando as lesões bucais que necessitam de avaliação na atenção secundária, documentos de referência permitem avaliar a necessidade clínica e a urgência de cada caso, com impactos na priorização do acesso. A falta de detalhamento e concisão dos documentos de referência impacta na agilidade do fluxo do paciente entre os níveis de atenção à saúde e, consequentemente, na qualidade do cuidado a ele oferecido. Visando aprimorar o detalhamento dos documentos de referência em Estomatologia na região da Grande Florianópolis, Santa Catarina, foi desenvolvido o OralDESC - um roteiro de apoio à descrição de lesões bucais. O roteiro também vem sendo utilizado pelas disciplinas relacionadas ao Diagnóstico Bucal na Universidade Federal de Santa Catarina. Além de apresentar o roteiro, este trabalho procura ampliar a análise da questão, apresentando alguns desafios para a implantação dos complexos regulatórios no Brasil, bem como as dificuldades relatadas no país e em outras
Professora do Departamento de Patologia e do Ambulatório de Estomatologia do Núcleo de Odontologia Hospitalar do Hospital Universitário, Universidade Federal de Santa Catarina

** Mestre em Odontologia, Doutoranda do Programa de Pós-Graduação em Odontologia, Universidade Federal de Santa Catarina

parte do mundo no que se refere à qualidade dos documentos de referência. Adicionalmente, apresenta a visão de alguns autores sobre o potencial educativo dos processos de referência e contrar-referência e a importância da formação para a comunicação escrita. São partilhadas, ainda, reflexões sobre a necessidade de inclusão, nos currículos acadêmicos, de atividades que permitam o desenvolvimento de habilidades de comunicação por estudantes de Odontologia, de forma a capacitá-los à atuação em redes de atenção que privilegiam a interação multiprofissional, interdisciplinar e transdisciplinar.

Descritores: Atenção Primária à Saúde. Atenção Secundária à Saúde. Guia. Medicina Bucal. Referência e Consulta.

\section{INTRODUÇÃO}

Os questionamentos que deram origem a este trabalho ocorreram a partir da observação de fatos - e da busca por estratégias para solucionar o impasse por eles estabelecido. As autoras estão vinculadas ao Ambulatório de 
Estomatologia do Núcleo de Odontologia Hospitalar $(\mathrm{AE} / \mathrm{NOH})$ - através do qual o Hospital Universitário da Universidade Federal de Santa Catarina (HU-UFSC) presta atendimento ambulatorial a pacientes portadores de lesões bucais. O AE/NOH atendeu pacientes por livre demanda de 1996 até agosto de 2010, quando foi inserido como unidade executante da Rede de Atenção à Saúde da região da Grande Florianópolis, nos níveis secundário e terciário de atenção ${ }^{1}$. A partir desse momento, o encaminhamento de pacientes passou a ser gerenciado pela Secretaria Municipal de Saúde, através do SISREG - sistema online disponibilizado pelo Ministério da Saúde. Neste modelo, os profissionais das Unidades Básicas de Saúde (UBS) registram a necessidade da consulta no sistema e, com base nas informações registradas, é agendada a consulta no nível secundário de atenção, determinando-se também a prioridade do acesso.

Após alguns meses de agendamento pelo novo modelo, a Central de Regulação procurou o $\mathrm{AE} / \mathrm{NOH}$ para estabelecer diretrizes para a priorização do acesso. Ao analisar as informações das solicitações de consultas, no entanto, percebeu-se que mesmo com um protocolo de regulação seria difícil proceder a priorização, já que muitos pedidos de consulta não forneciam a descrição detalhada das lesões ${ }^{1}$. A dificuldade em proceder tal descrição também vinha sendo observada entre estudantes do Curso de Graduação em Odontologia que desenvolviam atividades no AE/NOH. Buscando instrumentalizar o desenvolvimento dessa habilidade, as autoras desenvolveram um roteiro de apoio, denominado OralDESC.

Além de apresentar o roteiro, as autoras propõem a reflexão sobre a importância do desenvolvimento da comunicação escrita enquanto competência essencial à adequada troca de informações clínicas entre os diferen- tes níveis de atenção do Sistema Único de Saúde - SUS.

\section{O COMPLEXO REGULATÓRIO DO SUS E A REGULAÇÃO DE CONSULTAS EM ESTOMATOLOGIA}

A segmentação da atenção à saúde no SUS em diferentes níveis de atenção decorre da necessária racionalização do processo de trabalho, com vistas à potencialização dos recursos disponíveis. O fluxo de usuários entre os níveis gera uma demanda por serviços que necessita de ordenação. Denomina-se de regulação do acesso à assistência ou regulação assistencial o conjunto de relações, saberes, tecnologias e ações que intermediam tal demanda ${ }^{2}$. A ação regulatória envolve o processo de operacionalização, monitoramento e avaliação das solicitações de procedimentos, observando-se - além das questões clínicas protocolos estabelecidos para disponibilizar a alternativa assistencial mais adequada ${ }^{3}$.

O Ministério da Saúde vem fomentando a implantação de complexos reguladores, incluindo a informatização da regulação assistencial através de um aplicativo online denominado Sistema de Regulação (SISREG). A figura do regulador é chave no processo regulatório, estando sua atuação situada na interface entre a rotina clínica e a gestão dos serviços. O regulador tem acesso às evidências clínicas do caso através das informações disponíveis no pedido de consulta, e é com base nessas informações que decide o fluxo de um caso em específico ${ }^{3}$.

A articulação entre os níveis de atenção se dá por mecanismos de referência e contrarreferência, e a organização dos fluxos é estabelecida em uma rede hierarquizada e regionalizada ${ }^{3}$. A referência representa o encaminhamento para atendimento em serviços de maior densidade tecnológica (níveis secundário e/ou terciário de atenção) enquanto a con- 
trarreferência diz respeito ao encaminhamento para um nível de menor densidade tecnológica (frequentemente representado pela Unidade Básica de Saúde - UBS) ${ }^{4,5}$.

Há variações regionais nos processos de regulação, e a adesão ao SISREG tem sido gradativa $^{6}$. Segundo a Coordenação de Desenvolvimento de Sistemas de Saúde do Departamento de Informática do SUS ${ }^{7}$, estão implantadas no Brasil 204 centrais de regulação ambulatorial e 19 centrais de regulação hospitalar. Na região da Grande Florianópolis, Santa Catarina, o acesso ao nível secundário de atenção ocorre a partir das UBS, via SIS$\mathrm{REG}^{8}$. Para a regulação de consultas de pacientes portadores de lesões bucais, ofertar ao regulador a descrição detalhada das características da lesão (eventualmente associada a informações referentes a hábitos como tabagismo e etilismo) é fundamental para a priorização do acesso. O OralDESC foi desenvolvido para ser uma ferramenta de apoio a este processo de descrição.

\section{O ROTEIRO DE APOIO À DESCRI- ÇÃO DE LESÕES BUCAIS}

O OralDESC foi elaborado com base na literatura da área de Estomatologia e na experiência didática e clínica das autoras, assim como validado na forma de um piloto com cirurgiões-dentistas e estudantes de Odontologia para verificação da sua adequação.

É composto de duas partes: (1) uma sequência de itens descritivos, separados em seis seções: identificação da lesão/lesão fundamental, localização, características clínicas, comentários adicionais, hábitos comportamentais e tratamentos prévios (Figura 1); e (2) ilustrações, contemplando os seguintes itens descritivos: lesões fundamentais, tipos de inserção e características superficiais comumente encontradas em lesões bucais (Figura 2).

Foram acrescidas possibilidades de respostas aos itens descritivos, visando a uniformização de termos. As ilustrações oferecem suporte visual à escolha dos termos adequados. Nos itens 4, 5 e 6, foram incluídas perguntas que levem o usuário a fornecer maior detalhamento de dados de anamnese e de fatores que possam ter contribuído para a ocorrência da lesão.

\section{REVISÃO DA LITERATURA}

\subsection{Comunicação em saúde: os desafios dos processos de referência e contrarrefe- rência}

$\mathrm{Na}$ tentativa de compreender a origem e buscar soluções para o problema detectado, buscou-se na literatura relatos que enfocassem a comunicação em saúde, a implantação de complexos regulatórios e a construção de documentos de referência e contrarreferência.

Etimologicamente, comunicare (do latim) significa tornar comum. Vários são os modelos que buscam descrever o fenômeno da comunicação, sendo o mais básico o chamado Modelo de Lasswell, datado dos anos $40^{9}$. Este modelo descreve o ato da comunicação como as respostas às seguintes perguntas: Quem? Diz o que? Através de que canal? Para quem? Com que resultado? ("who says what to whom in what channel with what effect?"). A estas perguntas correspondem os seguintes elementos, considerados como básicos na comunicação: emissor, mensagem, meios, receptor e efeito.

Diversos autores posteriormente integraram conceitos adicionais a este modelo: Shannon e Weaver (1949, apud Pereira, 2007), por exemplo, identificaram o ruído como qualquer perturbação no processo de comunicação e De Fleur (1966, apud Pereira, 2007) introduziu o conceito do feedback (comparação dos significados entre a mensagem enviada e a recebida) ${ }^{10}$. 
1) Lesão Fundamental (ver figuras)

- Superficial

Mácula/ Mancha/ Placa

- Conteúdo Sólido:

Pápula/ Nódulo

- Conteúdo Líquido:

Fístula / Vesícula / Bolha / Pústula

- Com Perda Tecidual:

Erosão / Úlcera / Fissura

\section{2) Localização}

- Estrutura afetada (mucosa jugal, língua, gengiva, lábios...)

- Lado afetado (direito, esquerdo, bilateral)

- Arcada afetada (superior, inferior, ambas)

- Região afetada (anterior, média, posterior)

\section{3) Características}

- Tamanho ( $\mathrm{mm}$ ou cm, Altura x Largura)

- Cor

- Inserção (ver figuras)

- Superfície (ver figuras)

- Palpação (ósseo, fibroso, mole, flutuante)

- Tempo de evolução (há quantos dias, semanas, meses ou anos apareceu a lesão?)

- Tipo de crescimento (contínuo, aumenta e diminui, aumenta em período específico?)

- Dor (localizada/difusa) (espontânea/provocada) (passageira/contínua/intermitente) (leve/ moderada/intensa) (paroxística/pulsátil/queimação)

4) Observações adicionais da região da lesão, se pertinente:

- Paciente usuário de próteses? (nova/antiga, total/parcial)

- Lesão sai à raspagem?

- Possibilidade de trauma local (mordedura? prótese mal adaptada?)

5) Hábitos (especialmente lesões vermelhas ulceradas ou lesões brancas)

- Fumo:

1. Há quanto tempo?

2. Tipo de fumo (cigarro? charuto? cachimbo?)

3. Quantos cigarros/dia?

- Álcool:

1. Há quanto tempo?

2. Tipo de bebida (cerveja? cachaça? vinho?)

3. Frequência de consumo?

- Drogas ilícitas:

1. Tipo?

2. Há quanto tempo?

6) Tratamentos Prévios (se houve)

- Qual tratamento/medicação?

- Surtiu efeito?

Figura 1 - Sequência de itens descritivos do OralDESC. 

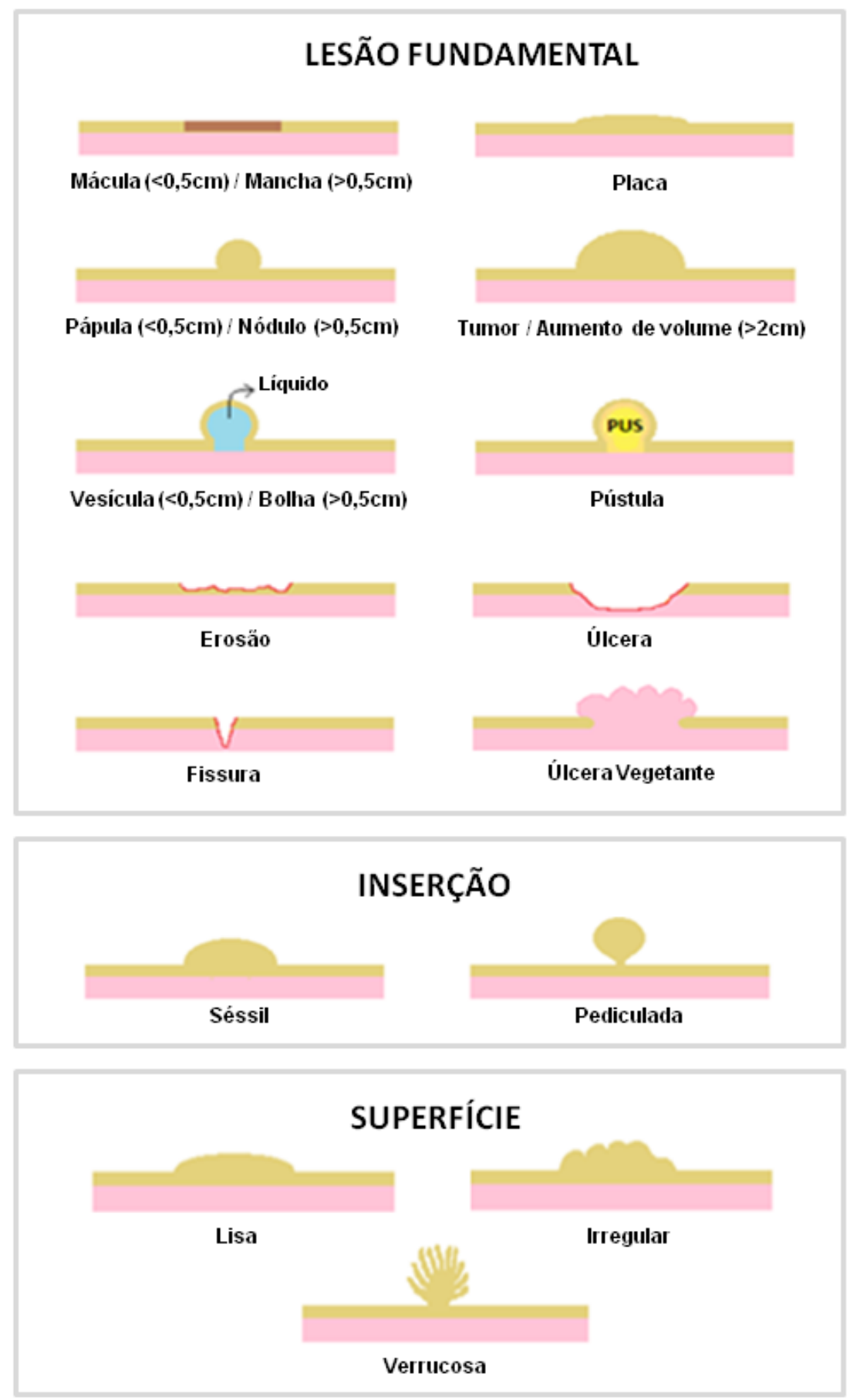

\section{LEGENDA $\quad$ Epitélio $\quad$ Conjuntivo}

Figura 2 - Figuras ilustrativas do OralDESC, contemplando aspectos das lesões fundamentais, tipo de inserção e aspectos superficiais comuns em lesões bucais.

Ainda, toda mensagem é composta de códigos (combinação de signos utilizados na transmissão de uma mensagem), muitas vezes bastante específicos; a comunicação só se concretiza se o receptor souber decodificar a mensagem $^{10}$.

Tais conceitos compõem a base de recentes sistemas de treinamento de equipes de 
saúde para o trabalho colaborativo, como é o caso do TeamSTEPPS ${ }^{\circledR}$ (Team Strategies and Tools to Enhance Performance and Patient Safety) ${ }^{11}$, desenvolvido pela US Agency for Healthcare Research and Quality em colaboração com o Departamento de Defesa norteamericano $^{12}$. Este modelo defende que a efetividade na comunicação em uma equipe de saúde depende basicamente de quatro pontos:

(1) Completude: oferecer todas as informações relevantes;

(2) Clareza, em grande parte dependente do uso de uma terminologia padrão;

(3) Concisão, pois detalhes desnecessários podem gerar confusão;

(4) Oportunidade: feita a tempo, pois o atraso no repasse da informação pode comprometer a situação do paciente.

Num sistema de saúde em que o paciente transita por diferentes níveis de atenção e/ou necessita de avaliação interdisciplinar ou multiprofissional, a troca de informações é essencial para garantir que o cuidado ocorra de forma adequada e em tempo oportuno. Documentos de referência e contrarreferência são meios comuns pelos quais essa comunicação se estabelece ${ }^{3,13-16}$.

François ${ }^{17}$ defende que "boa comunicação é essencial para consultas e referências seguras e de qualidade". No Canadá, o autor relata haver pouco contato entre os médicos da atenção primária e especialistas; assim, comunicações por escrito são o meio mais comum - e às vezes o único - para troca de informações. Para o autor, a comunicação insuficiente se reflete em "efeitos adversos" como atraso no diagnóstico, repetição desnecessária de exames e aumento na polimedicação, o que entre outras consequências - potencialmente aumenta os custos dos tratamentos de saúde. Tattersal ${ }^{13}$ acrescentou que a boa comunicação evitaria ainda a insatisfação do paciente e a perda de confiança do mesmo no generalista.
Há referências, na literatura, à dificuldade de comunicação entre profissionais da saúde no processo de referência, sendo classificada como inadequada em termos de qualidade $^{18-20}$, tempo ${ }^{18}$ e conteúdo ${ }^{20}$.

Ferreira et al. ${ }^{21}$ avaliaram a implantação do Complexo Regulador no Sistema Público de Saúde de Ribeirão Preto, São Paulo, Brasil, e identificaram a informação clínica incompleta como o principal problema para a ação regulatória de consultas médicas. Segundo os autores, "quando o caso clínico era bem descrito, o trabalho dos reguladores era facilitado, além de permitir a identificação de situações que poderiam ser atendidas pela atenção básica, e não primariamente encaminhadas".

Harris et al. ${ }^{22}$ estudaram o processo de contrarreferência no município de Camaragibe, Pernambuco, Brasil, ao entrevistar dez médicos das especialidades de neurologia, obstetrícia, ginecologia, psiquiatria, otorrinolaringologia e colposcopia. Apesar da maioria dos especialistas compreender a importância da contrarreferência para a continuidade do cuidado, relataram não a enviar rotineiramente. Entre os motivos para tal, destacaram: inadequada organização do espaço de trabalho; falta de tempo, recursos e materiais; inibição para a comunicação; isolamento profissional; e a própria educação médica recebida pelos participantes, a qual não enfatizava aspectos de saúde pública. Os autores recomendaram a reavaliação dos currículos acadêmicos de forma a refletir a importância de um sistema integrado.

Almeida et al. ${ }^{6}$ descreveram a integração dos serviços de saúde em Belo Horizonte, Florianópolis e Vitória, municípios brasileiros que já haviam implantado centrais de regulação gerenciadas pelo SISREG. Os autores afirmaram que instrumentos de referência e contrarreferência representam uma importante ferramenta para a integração. Relataram 
a existência de mecanismos formais para referência e contrarreferência; no entanto, a maior parte dos gestores afirmou que a contrarreferência não é prática comum, apesar de os fluxos estarem instituídos. Tal informação foi confirmada pelos baixos percentuais de médicos que afirmaram recebê-la sempre ou na maioria das vezes, variando de $2,8 \%$ em Belo Horizonte a 11,5\% em Florianópolis. Os autores defendem que a avaliação sistemática dos encaminhamentos pode qualificar as referências e promover a utilização racional dos recursos especializados. Avaliam que a ausência da contrarreferência sugere necessidade de maior integração, e que esforços devem ser empreendidos junto aos profissionais da rede para garantir a continuidade informacional.

Machado, Colomé e Beck ${ }^{23}$ analisaram a percepção de profisssionais da Estratégia de Saúde da Família acerca do sistema de referência e contrarreferência de um município do interior do Rio Grande do Sul, evidenciando lacunas de comunicação entre profissionais dos diferentes níveis de atenção.

Para Fratini, Saupe e Massaroli ${ }^{24}$, referência e contrarreferência são fundamentais para a efetivação dos princípios do SUS; no entanto, afirmaram que estes processos ainda se encontram num estágio de pouco desenvolvimento, tanto em relação aos seus possíveis sentidos teóricos quanto no que refere à efetivação e divulgação de experiências, exitosas ou não. Estes mesmos autores afirmaram:

“... a busca por mecanismos facilitadores do estabelecimento de processos de referência e contrarreferência pode ser considerada fundamental para a concretização do princípio da integralidade; mas é evidente também que as experiências para viabilizar este modelo técnico-assistencial ainda são muito isoladas e frágeis, não permitindo generalizações, mesmo ao nível de políticas públicas municipais".
O processo de referência e contrarreferência parece não ser problema apenas no Brasil. Na Noruega, Farup et al. ${ }^{25}$ abordaram a relação entre a inadequada interação dos serviços de saúde e a falta de resolutividade dos sintomas de pacientes com refluxo gastroesofágico (RGE). Para tal, entrevistaram 20 pacientes portadores de RGE com queixas persistentes, questionando-os quanto à possível causa da falha no tratamento. As entrevistas foram anonimizadas, transcritas e avaliadas por 18 médicos (6 generalistas, 6 gastroenterologistas e 6 cirurgiões gástricos), aos quais foi solicitado que atribuíssem a responsabilidade pela falha na resolutividade: (i) ao paciente, (ii) ao generalista, (iii) ao especialista ou (iv) à interação entre os serviços de saúde. Falha de interação foi apontada como a causa mais importante (35\%), seguida por falha do cuidado primário pelo generalista $(28 \%)$, do cuidado secundário pelo especialista $(27 \%) \mathrm{e}$ da falha do paciente (10\%).

Lin $^{16}$ relatou evidências, nos Estados Unidos, de transferência de informação insuficiente, com falta de comunicação no sentido generalista $\rightarrow$ especialista em $50 \%$ das referências, e no sentido especialista $\rightarrow$ generalista em $45 \%$ dos casos. No estudo, mais de $70 \%$ dos especialistas classificaram as informações recebidas como regulares ou insatisfatórias.

Para O’Byrne et al. ${ }^{26}$, documentos de referência deveriam ser suficientemente completos e acurados para assegurar um sistema seguro e efetivo; ainda, informações deveriam ser fornecidas de forma consistente por diferentes generalistas em diferentes regiões, independente do sistema de referência utilizado.

Foot, Naylor e Imison $^{27}$ listaram os elementos que qualificariam referências de alta qualidade: 1) necessidade: pacientes referenciados apenas quando necessário e sem perda de tempo; 2) destino: local apropriado; 3) condução do processo de referência em si, 
com documentos de referência contendo as informações necessárias e em um formato acessível ao destinatário. Finalmente, pontuaram que boa a comunicação entre generalistas e especialistas deve ser incentivada, já que facilita a troca de informações e oferece oportunidades de aprendizado, servindo de base para diagnósticos e referências de alta qualidade.

O Grupo Europeu de Trabalho na Qualidade da Prática Familiar afirmou que para haver melhora efetiva na interface entre a comunicação, o cuidado contínuo do paciente e a continuidade do cuidado, são necessárias mudanças não somente nos sistemas de saúde, mas também no modo como os profissionais encaram seu papel nesse contexto ${ }^{28}$.

Machado, Colomé e Beck ${ }^{23}$ defendem ser necessário fortalecer a formação dos profissionais da saúde, de forma que sejam capazes de compartilhar saberes e decisões, ampliando e potencializando sua capacidade crítica e de intervenção na realidade. Segundo as autoras, esses são fatores essenciais para a sistematização e efetivação dos processos de referência e contrarreferência.

\subsection{O potencial educativo do processo de referência e contrarreferência e o treina- mento para o desenvolvimento de habili- dades de comunicação escrita}

"A referência é o método mais inexplorado para influenciar as atitudes dos consultores. A contrarreferência é a rota mais negligenciada para a educação dos generalistas."

(Pringle, 1991 apud Tattersall et al.,

A comunicação entre profissionais com diferentes conhecimentos, experiências, habilidades e expertises funciona como aprendizado mútuo ${ }^{29,30}$. Em 1990, Westerman et al. ${ }^{30}$ já ressaltavam o potencial educativo de documentos de referência e contrarreferência.
Para estes autores, generalistas e especialistas têm muito a ensinar um ao outro, e quando os documentos de referência e contrarreferência são pobres em dados, a oportunidade de aprendizado se perde.

Smith e Khutoane ${ }^{31}$, estudando a falta de documentos de contrarreferência na África do Sul, relataram que os médicos da atenção secundária entendiam seu papel no sistema de saúde apenas como supervisores de residentes. Desestimulados por documentos de referência de baixa qualidade, referiram um sentimento de "futilidade" ao escrever contrarreferências. Não foi considerado por eles o possível impacto educacional das contrarreferências para residentes e generalistas, assim como a importância da rede (networking) enquanto troca de saberes.

Para Lingard et al..$^{32}$, quando um profissional decide filtrar os dados do paciente $\mathrm{e}$ escolhe expressá-los de uma forma particular, condiciona pensamentos e ações de outros profissionais. Deste ponto de vista, a linguagem traria em si não apenas uma força descritiva, mas também uma força construtiva. A linguagem, em virtude da sua função social, embute valores, incorporando crenças de uma dada coletividade; assim, atitudes direcionadas a pacientes e outros profissionais acabam sendo refletidas e reproduzidas nas características das instituições. A conexão linguagem/ideias seria fundamental: palavras modelam percepções e atitudes - e as práticas a elas associadas. Considerando a comunicação interprofissional, uma das habilidades que o profissional de saúde deveria desenvolver é a capacidade de produzir textos com informações relevantes; para tanto, precisa desenvolver o senso lógico que o permita ressaltar, dentro de um conjunto variável de dados sobre o paciente, apenas aqueles relevantes ao contexto clínico específico. Citando um trabalho anterior $^{33}$, os autores afirmaram que documentos de referência refletem conclusões dos 
escritores, expectativas sobre os leitores e regulações do sistema em si. O conhecimento clínico seria apenas um elemento neste contexto biomédico, e a relevância de um documento de referência seria determinada tanto pelo processo do raciocínio diagnóstico (relevância clínica) quanto pelo processo profissional da emissão de opinião fundamentada em grupos multiprofissionais (relevância retórica). Os autores ressaltaram de forma incisiva a importância da formação no processo de desenvolvimento das habilidades de comunicação escrita quando afirmaram:

"Uma abordagem casual para educar aprendizes acerca do conceito de relevância [da comunicação em saúde] incorre em risco significativo no amplo contexto do cuidado efetivo ao paciente, pois se documentos de referência forem percebidos como irrelevantes, podem frustrar ou confundir seus destinatários, o que por sua vez pode ameaçar o trânsito seguro do paciente através do sistema de saúde."

Outros autores avaliaram o impacto do treinamento para escrita de documentos de referência e contrarreferência. Nestel e Kidd ${ }^{34}$, por exemplo, avaliaram a viabilidade de sessões de ensino para estudantes do segundo ano de Medicina visando sensibilizá-los para a comunicação escrita no contexto dos cuidados em saúde centrados no paciente, bem como auxiliá-los no desenvolvimento das habilidades relacionadas. Na experiência das autoras, tais sessões foram positivas, já que, após quatro meses, os estudantes que assistiram às sessões reportaram mais habilidade para a tarefa do que aqueles que não assistiram. Os participantes identificaram possíveis fontes de problemas, como potenciais "mal-entendidos" com o uso de abreviações, e as implicações de registros incorretos e informações incompletas. As autoras ponderaram que o melhor momento para essas sessões talvez fosse aquele imediatamente anterior a experiências clínicas. Atividades subsequentes, integradas a um ambiente real, permitiriam a completude do desenvolvimento dessa habilidade. As autoras finalizaram ponderando que talvez fosse interessante estender esse perfil de atividade aos níveis de educação médica continuada e a outros grupos profissionais, incluindo ambientes multiprofissionais - onde o significado da comunicação em saúde se amplia.

Tattersall et al. ${ }^{13}$ desenvolveram um curso de treinamento para oncologistas para qualificá-los para a comunicação com generalistas após a consulta do paciente. Os autores concluíram que o treinamento melhorou significativamente alguns itens considerados importantes nos documentos de contrarreferência, como relatos, desejos e expectativas dos pacientes, detalhes psicossociais, e como/quando contatar o oncologista.

$\mathrm{Na}$ área de Odontologia, não foram encontrados relatos que descrevessem algum tipo de treinamento para a elaboração de documentos de referência ou contrarreferência.

\section{DISCUSSÃO}

O modelo brasileiro de atenção à saúde é essencialmente colaborativo, e se estrutura em redes ${ }^{35}$, onde serviços e sistemas de saúde se articulam. Nesse modelo, a qualidade de referências e contrarreferências é fundamental $^{17,24}$ e a colaboração essencial, já que diferentes atores assumem diferentes papéis, dividindo responsabilidades na busca de soluções para os problemas de saúde dos pacientes. Por sua vez, a colaboração depende da boa comunicação.

Documentos de referência e contrarreferência são registros escritos de pensamentos ou informações, sendo ferramentas de comunicação entre profissionais de saúde que desenvolvem um trabalho colaborativo $^{13,17,21,26}$. No caso específico da regulação em Estomatologia na região da 
Grande Florianópolis, a falta de informações em documentos de referência em Estomatologia impactou na falta de resolutividade do sistema, pela necessidade de retorno do pedido de consulta para inclusão de informações adicionais ${ }^{1}$. O OralDESC foi criado para ser uma ferramenta de apoio aos cirurgiões-dentistas das UBS do município de Florianópolis, mas também para uso nas atividades do Curso de Graduação em Odontologia e do AE/NOH. Estudos estão em andamento para avaliar a adequação do roteiro, tanto do ponto de vista da autopercepção dos usuários quanto do seu potencial para melhorar a descrição de lesões bucais. Ressalta-se, ainda, a necessidade do desenvolvimento de estudos sobre a efetividade do instrumento, tanto no que se refere à qualificação das descrições de lesões bucais quanto ao seu eventual impacto na comunicação interprofissional e no processo de referência em Estomatologia.

Acredita-se que o OralDESC tenha potencial para auxiliar os usuários na descrição de lesões bucais considerando os três primeiros pontos destacados pelo TeamSTEPPS $^{\circledR}{ }^{11}$, e em particular no que se refere ao estabelecimento de terminologiapadrão, que permitiria o fornecimento das informações necessárias e em um formato consistente, independente de indivíduo ou região ${ }^{13,26}$. Embora a terminologia seja conhecida, frequentemente há dificuldade, entre estudantes de Odontologia e cirurgiõesdentistas, de transferir esse conhecimento para as atividades clínicas. Por esse motivo, optouse por acrescentar ilustrações dos termos que, na experiência das autoras, geram mais dúvidas e questionamentos na prática clínica.

O quarto ponto do TeamSTEPPS ${ }^{\circledR 11}$ a oportunidade da comunicação - está mais na dependência do profissional que referencia e do sistema em si, embora haja a expectativa de - com o uso do roteiro - haver redução no número de solicitações de consulta devolvidas ${ }^{1}$.

Cabe ressaltar que o processo de comunicação em saúde é complexo, sendo necessário identificar outros pontos que reduzam a resolutividade do sistema. O OralDESC contempla apenas documentos de referência, e atenção semelhante deve ser dada ao processo de contrarreferência, especialmente considerando as possibilidades de aprendizado embutidas na interação entre os níveis de atenção $^{13,29-32}$.

\section{CONSIDERAÇÕES FINAIS}

A formação de profissionais para atuar em sistemas de saúde como o SUS é tarefa complexa, e deve contemplar tanto as necessidades dos usuários quanto do sistema. A interface ensino-serviço potencializa a observação dos desafios regionais na assistência à saúde, cabendo também às Universidades, através de seus programas de pós-graduação, a busca de soluções para as dificuldades observadas, em parceria com a rede de atenção e seus gestores. Finalmente, ressalta-se que as Diretrizes Curriculares Nacionais em Odontologia preveem a formação para atuação multiprofissional, interdisciplinar e transdisciplinar, e a formação odontológica precisa envolver ações que redundem na atuação profissional desejada.

Embora a comunicação escrita seja uma competência essencial para a comunicação clínica ${ }^{15,17,32,34}$, o desenvolvimento dessa habilidade não parece estar formalmente integrado à maioria dos currículos acadêmicos no Brasil. A literatura revisada pelas autoras - e apenas parcialmente contemplada neste artigo - permitiu perceber a amplitude e profundidade das discussões em andamento no que se refere aos processos de comunicação em saúde - e que incluem também o desenvolvimento de habilidades de comunicação com pacientes $^{36,37}$ e corpo técnico ${ }^{38}$. Tal conjunto 
de reflexões merece entrar na pauta de discussões de instituições comprometidas com a qualificação do ensino, como a Associação Brasileira de Ensino Odontológico (ABENO), bem como de iniciativas institucionais para a formação docente - incluindo a formação docente nos programas de pós-graduação.

\section{REFERÊNCIAS}

1. Rohden NT, Costa AM, Silva MLB. Análise sobre a inserção da especialidade de Estomatologia do Hospital Universitário no Sistema de Regulação de Florianópolis e seus benefícios na prática odontológica. Coleção Gestão da Saúde Pública. 2013;13:25-45. Disponível em: http:// gsp.cursoscad.ufsc.br/wp/wp-content/uplo ads/2013/03/Volume-13-Artigo02.pdf

2. O SUS de A a Z. Brasília; 2009; Série F. Comunicação e Educação em Saúde:481. Disponível em: http://bvsms.saúde.gov.br/ bvs/publicacoes/sus_az_garantindo_saude _municipios_3ed_p1.pdf

3. BRASIL. Ministério da Saúde. Diretrizes para a implantação de Complexos Reguladores [Internet]. Brasília; 2006. Disponível em: http://bvsms.saude.gov. br/bvs/ publicacoes/DiretrizesImplantComplexos Reg2811.pdf

4. Conselho Nacional de Secretários de Saúde. Atenção Primária e Promoção da Saúde [Internet]. Conselho Nacional de Secretários de Saúde, editor. Brasília; 2011. 1 p. Disponível em: http:// www.conass.org. br/bibliotecav3/pdfs/colecao2011/livro_3.pdf

5. BRASIL. Ministério da Saúde. Gestão financeira do Sistema Único de Saúde [Internet]. 3rd ed. (null), editor. Brasília: Ministério da Saúde; 2003. Disponível em: http://www.fns2.saude.gov. br/documentos/Publicacoes/Manual_Gestao Fin SUS.pdf
6. Almeida PF de, Giovanella L, Mendonça MHM de, Escorel S. Desafios à coordenação dos cuidados em saúde: estratégias de integração entre níveis assistenciais em grandes centros urbanos. Cad Saude $\mathrm{Pu}$ blica. Escola Nacional de Saúde Pública, Fundação Oswaldo Cruz; 2010;26(2) :286-98. Disponível em: http://www. scielo.br/pdf/csp/v26n2/08.pdf

7. BRASIL. SISREG - Sistema Nacional de Regulação [Internet]. [cited 2015 Jul 21]. Disponível em: http://www2. datasus.gov.br/DATASUS/index.php?acao= $11 \& i d=30430$

8. Bender ADS, Molina LR, Mello ALSF de. Absenteísmo na atenção secundária e suas implicações na atenção básica. Revista Espaço para a Saúde. Londrina; 2010;11(2):56-65. Disponível em: http:// www.uel.br/ccs/espacoparasaude/v11n2 labsent.pdf

9. Lasswell HD. The structure and function of communication in society. In: Bryson L, editor. The Communication of Ideas. New York; 1948

10.Pereira DC. A relação espírito-sociedade. Nova Educação na Nova Ciência para a Nova Sociedade. Cidade do Porto: Universidade do Porto; 2007. pp. 401-86.

11.Agency for Healthcare Research \& Quality. TeamSTEPPS ${ }^{\circledR}$ : Strategies and Tools to Enhance Performance and Patient Safety [Internet]. Disponível em: http://www. ahrq.gov/professionals/education/curriculum-tools/teamstepps/ index.html

12.Alonso A, Baker DP, Holtzman A, Day R, King H, Toomey L, et al. Reducing medical error in the Military Health System: How can team training help? Human Resource Management Review. 2006;16(3) :396-415. Disponível em: http://www. sciencedirect.com/science/article/pii/S105 3482206000520 
13.Tattersall MHN, Butow PN, Brown JE, Thompson JF. Improving doctors' letters. Med J Aust. 2002 Nov 4;177(9) :516-20. Disponível em: https://www. mja.com. au/system/files/issues/177_09_041102/ tat10851_fm.pdf

14.Berta W, Barnsley J, Bloom J, Cockerill R, Davis D, Jaakkimainen L, et al. Enhancing continuity of information - essential components of a referral document. Can Fam Physician. The College of Family Physicians of Canada; 2008 Oct 1;54(10):14326. Disponível em: http:// www.cfp.ca/content/54/10/ 1432.full

15.Chetcuti K, Farrugia R, Cassar K. GP referral letters: time for a template? Malta Medical Journal; 2009 Jun;21 (2):26-9. Disponível em: https://www. um.edu.mt/ library/oar/handle/123456789/921

16.Lin CY. Improving care coordination in the specialty referral process between primary and specialty care. N C Med J. 2012 Jan;73(1):61-2. Diponível em: http://nciom.org/wp-content/uploa ds/2012/01/73115-web.pdf

17.François J. Tool to assess the quality of consultation and referral request letters in family medicine. Can Fam Physician. 2011 May;57(5):574-5. Disponível em: http://www.cfp.ca/content/57/5/574.full. pdf + html

18.Gandhi TK, Sittig DF, Franklin M, Sussman AJ, Fairchild DG, Bates DW. Communication breakdown in the outpatient referral process. J Gen Intern Med. Springer; 2000 Sep;15(9):626-31. Disponível em: http://www.ncbi. nlm.nih.gov/pmc/ articles/PMC1495590/pdf/jgi 91119.pdf

19.Hodgson TA, Buchanan JAG, Garg A, Ilyas SE, Porter SR. An audit of the UK national cancer referral guidelines for suspected oral mucosal malignancy. Br Dent J. 2006 Nov 25;201(10):643-7. Disponível em: http://www.nature. com/ bdj/journal/v201/n10/pdf/4814262a.pdf

20.Ibiyemi O, Ibiyemi T. Quality and contents of referral letters from peripheral health centers to the dental centre of a teaching hospital, southwestern Nigeria. Acta odontologica Scandinavica. Informa Healthcare Stockholm; 2012 Mar;70(2) :165-8. Disponível em: http://informa healthcare.com/doi/pdf/10.3109/000163 $\underline{57.2011 .600712}$

21.Ferreira JBB, Mishima SM, Santos JSD, Forster AC, Ferraz CA. O complexo regulador da assistência à saúde na perspectiva de seus sujeitos operadores. Interface (Botucatu). Interface - Comunicação, Saúde, Educação; 2010;14(33):345-58. Disponível em: http://www.scielo.br/pdf/icse/v14 n33/a09v14n33.pdf

22.Harris M, Ferreira A, Morães I, de Andrade F, de Souza D. Reply letter utilization by secondary level specialists in a municipality in Brazil: a qualitative study. Rev Panam Salud Publica. 2007 Feb;21(2-3):96110. Disponível em: http://www. scielosp.org/pdf/rpsp/v21n2-3/05.pdf

23.Machado LM, Colomé JS, Beck CLC. Estratégia de Saúde da Família e o sistema de referência e de contra-referência: um desafio a ser enfrentado. Revista de Enfermagem da UFSM [Internet]. 2011 Jan 21;1(1):31-40. Disponível em: http:// cascavel.ufsm.br/revistas/ojs-2.2.2/index.php/ reufsm/article/view/2337

24.Fratini JRG, Saupe R, Massaroli A. Referência e contra referência: contribuição para a integralidade em saúde - DOI: 10.4025/cienccuidsaude.v7i1. 4908. Cienc Cuid Saude. 2008 Sep 8;7(1):65-72. Disponível em: http://eduem.uem.br /ojs/index.php/CiencCuidSaude/article/download/4908/3211

25.Farup PG, Blix I, Førre S, Johnsen G, 
Lange O, Johannessen $\mathrm{R}$, et al. What causes treatment failure - the patient, primary care, secondary care or inadequate interaction in the health services? BMC Health Serv Res. BioMed Central Ltd; 2011;11(1):111. Disponível em: http:// www.biomedcentral.com/content/pdf/ 1472-6963-11-111.pdf

26.O'Byrne L, Darlow C, Roberts N, Wilson G, Partridge MR. Smoothing the passage of patients from primary care to specialist respiratory opinion. Prim Care Respir J. 2010 Sep;19(3):248-53. Disponível em: http://www.nature.com/articles/pcrj $\underline{201028}$

27.Foot C, Naylor C, Imison C. The quality of GP diagnosis and referral [Internet]. Report - The King's Fundation. 2010 p. 77. Disponível em: http://www. kingsfund.org.uk/sites/files/kf/Diagno sis $\% 20$ and\%20referral.pdf

28.Kvamme OJ, Olesen F, Samuelson M, Samuelsson M. Improving the interface between primary and secondary care: a statement from the European Working Party on Quality in Family Practice (EQuiP). Qual Health Care. BMJ Group; 2001 Mar;10(1):33-9. Disponível em: http://qualitysafety.bmj.com/content/10/1 33.full.pdf+html

29. Newton J, Eccles M, Hutchinson A. Communication between general practitioners and consultants: what should their letters contain? BMJ [Internet]. BMJ Group; 1992 Mar 28;304(6830): 821-4. Disponível em: http://www.ncbi.nlm.nih.gov/pmc/articles /PMC1881665/pdf/bmj00066-0039.pdf

30.Westerman RF, Hull FM, Bezemer PD, Gort G. A study of communication between general practitioners and specialists. Br J Gen Pract. 1990 Nov;40(340): 445-9. Disponível em: http://www. ncbi.nlm.nih.gov/pmc/articles/PMC 1371413/pdf/brjgenprac00074-0007.pdf
31.Smith S, Khutoane G. Why doctors do not answer referral letters. South African Family Practice. Medpharm Publications Pty Ltd; 2009;51(1):64-7. Disponível em: http://www.tandfonline.com/doi/pdf/10.10 $\underline{\text { 80/20786204.2009.10873810 }}$

32.Lingard L, Hodges B, MacRae H, Freeman R. Expert and trainee determinations of rhetorical relevance in referral and consultation letters. Med Educ. 2004 Feb;38(2):168-76. Disponível em: http:// onlinelibrary.wiley.com/doi/10.1111/j.136 52923.2004. 01745.x/pdf

33.Lingard LA, Haber RJ. What do we mean by "relevance?" A clinical and rhetorical definition with implications for teaching and learning the case-presentation format. Acad Med. 1999 Oct;74(10 Suppl):S1247. Disponível em: http://journals. lww.com/academicmedicine/pages/articleviewer. aspx ?year $=1999 \&$ issue $=10000$ $\underline{\text { \&article }}=00061 \&$ type $=$ abstract

34.Nestel D, Kidd J. Teaching and learning about written communications in a United Kingdom medical school. Educ Health (Abingdon). 2004 Mar;17(1):27-34.

35.BRASIL. Ministério da Saúde. Portaria N ${ }^{\circ}$ 4.270, de 30 de dezembro de 2010. [Internet]. Dec 30, 2010. Disponível em: http://conselho.saude.gov.br/ultimas noticias/2011/img/07_jan_portaria4279 301210.pdf

36.Carey JA, Madill A, Manogue M. Communications skills in dental education: a systematic research review. European Journal of Dental Education. Blackwell Publishing Ltd; 2010 May;14(2):69-78. Disponível em: http://onlinelibrary.wiley.com/doi/ $\underline{10.1111 / \mathrm{j} .16000579 .2009 .00586 . \mathrm{x} / \mathrm{pdf}}$

37.Candlin S. Therapeutic communication. French Forest: Pearson Education Australia; 2008. $261 \mathrm{p}$.

38.Evans J, Henderson A, Johnson N. The future of education and training in dental 
technology: designing a dental curriculum that facilitates teamwork across the oral health professions. Br Dent J. Nature Publishing Group; 2010 Mar 13;208(5):22730. Disponível em: http://www.nature .com/bdj/journal/v208/n5/pdf/sj.bdj. 2010.208.pdf

\section{ABSTRACT \\ Proposal for a guideline for the description of oral lesions as a support tool for profes- sional communication in Oral Medicine}

Good written communication is an essential skill for the exchange of information between health professionals. In the context of Brazilian National Health Service, this exchange occurs via referral and reply letters. With regard to oral lesions that require evaluation in secondary care, referral letters allow the assessment of clinical need and the urgency for consultation with the oral health specialist. These factors determine the access priority in the secondary health system. The lack of details and conciseness of the referral letters impacts the patient flow throughout the healthcare levels and hence the quality of healthcare services. In order to improve the quality of information of the referral letters to specialists in Oral Medicine in the Greater Florianopolis city, Santa Catarina, Brazil, a guideline for supporting the description of oral lesions - the OralDESC - has been developed. The guideline is being used in the courses related to oral diagnosis consultations at the Federal University of Santa Catarina. This paper extends the analysis of the problem, discussing some challenges for the implementation of the referral procedures in Brazil as well as the difficulties reported in the country and in other countries regarding the quality of referral letters. Additionally, the paper provides views of some authors on the educational potential of referral and reply letters and the necessity of training health students for written communication. The authors share some thoughts about the need for inclusion of activities in the Brazilian Dentistry curricula aiming at developing communication skills in the students so as to prepare them to work in multidisciplinary, interdisciplinary and transdisciplinary teams.

Descriptors: Primary Health Care. Secondary Care. Guideline. Oral Medicine. Referral and Consultation.

Correspondência para:

Profa. Dra. Maria Inês Meurer

e-mail: meurer.m.i@ufsc.br

Universidade Federal de Santa Catarina

Campus Universitário - Trindade

88040-370 - Florianópolis - SC - Brasil 